\title{
Evidential significance of multiple fracture patterns on the glass in forensic ballistics
}

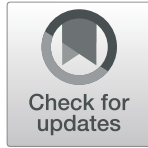

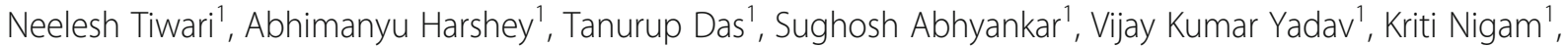
Vijay Raj Anand ${ }^{2}$ and Ankit Srivastava ${ }^{1 *}$ (i)

\begin{abstract}
Background: In the current scenario, glass plays a vital contribution in our standard everyday life. Fractured and fragmented glasses are most commonly encountered at the crime scene. During the commission of the crime, glass breaks and shatters into fragments or into minute particles that serve as trace evidence and may provide a significant lead for the probe. The fracture of glass could be the consequence of an intentional act or unintentional act. For the forensic purpose, study of glass fractures, i.e., fractography has paramount importance as it can provide noteworthy data and encourages an assortment of assessments to investigate the case especially in the shooting cases where a firearm is used. Multiple fractures on a glass pane also have enough potential to establish an investigative link. Presently, air guns are being used in many of the cases due to their simple accessibility. There are a number of cases that have been reported that involved the use of air guns.

Result: This study was conducted to evaluate the pattern of multiple fractures on the glass by the air rifle. For this purpose, glass panes of various thicknesses were test fired perpendicularly at a fixed range with an air rifle having round nose pellet. Measurements and analysis were made on some fixed parameter. Data was analyzed graphically and statistically to check the consistency in the trends.
\end{abstract}

Conclusion: Consistency in the trends was observed, which is analyzed by the test for the goodness of fit. The findings of this work have provided some significant different perspectives that may effectively contribute to the criminal investigation.

Keywords: Trace evidence, Glass fracture, Forensics, Fractography, Multiple fractures, Air rifle, Fracture pattern

\section{Background}

Glass is a supercooled mixture of the metallic silicates. Generally, glass is referred to as transparent, semitransparent, non-crystalline, and amorphous solid having number of physical properties, such as durability, rigidity, refractive index, and density (Copley 2001). These physical properties, especially refractive index, provide means of investigation for the forensic purpose (Jauhari et al. 1974; Stoney and Thornton 1985; Gogotsi and Mudrik 2010). Glass or glass fragments serve as physical evidence of a great value for the investigation of many offenses such as the burglary, arson, hit and run cases, shooting, and assault. A large variety of physical evidences is included under the term "Trace Evidence".

\footnotetext{
*Correspondence: ankit_forensic81@rediffmail.com

${ }^{1}$ Dr. A.P.J. Abdul Kalam Institute of Forensic Science and Criminology,

Bundelkhand University, Jhansi, Uttar Pradesh 284128, India

Full list of author information is available at the end of the article
}

Locard's Principle of Exchange is the fundamental concept in the production of physical and trace evidence (Caddy 2001; Robertson and Roux 2010; Mistek et al. 2018).

The analysis of glass fracture has been a subject matter of interest to the forensic community for a long time as it enables the investigator to establish lots of considerations such as cause of fracture, direction of impact, and angle of impact. The term "Fractography" represents the study of surface fracture to identify the mechanism of such fracture (Caddy 2001; Harshey et al. 2017).

Many eminent researchers gave their efforts to explain the mechanism of the fracture. Griffith echoed that fracture starts from the previous flaws known as Griffith flaw's (Griffith 1920). Law of Griffith was further extended as dynamic fracture mechanism by Mott (1964). Theoretically, the strength of any material is defined in terms of inter-atomic bond. Tensile stress depends upon 
various factors such as condition and size of surface, duration of load, and environmental conditions (Overend et al. 2007). As the projectile hits the glass pane, cracks are created due to the transfer of projectile's energy. Cracks propagates through the path of least resistance. The energy shock waves cause specific damage to the glass, originated from the point of impact (Grady 2010). In the context of forensic glass fracture analysis, the aspect of energy loss was experimentally demonstrated by Waghmare et al. (2003).

When any projectile, e.g., bullet or stone, hits the glass surface, stretching and compression occur. As the limit of tensile stress is crossed, it results in the breaking of the glass. The impact of the bullet or stone causes two special types of fractures, namely termed as radial and concentric (also known as spiral) fractures as demonstrated in Fig. 1. As the high velocity projectile penetrates the glass pane, there is a formation of a crater-shaped hole that is termed as cone fracture. The appearance of the cone may be observed at wide exit side of the hole (Mcjijnkins and Thornton 1973). In this context, the shape of the chip pattern (also called mist zone) around the hole is indicative of the direction of impact (Harshey et al. 2017). Hackle marks, stress marks, mirror zone, etc., are some other phenomena that may be observed in the fractured glass pane (Caddy 2001). These characters often provide a significant help to the investigator in the investigation of variety of offenses.

In this study, the soda-lime glass was taken. Soda-lime glass majorly contributes in the manufacture of windowpanes (flat glass), containers, etc. Sand $\left(\mathrm{SiO}_{2}, 63-74 \%\right)$, soda ash $\left(\mathrm{Na}_{2} \mathrm{CO}_{3}, 12-16 \%\right)$, and limestone $(\mathrm{CaO}, 7-$ $14 \%)$ contributes as raw material in the manufacturing of soda lime glass (Curran et al. 2000).

No chemical reaction is used in the air gun and the mechanism of air guns involves the mechanically pressurized air or other means. When the trigger is released, compressed air is let off into the barrel, pushing along a

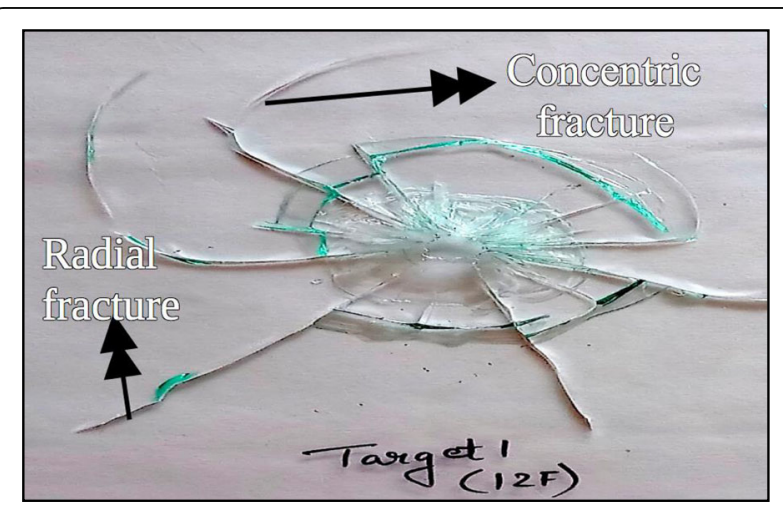

Fig. 1 Radial and concentric fractures specially made slug or pellet in front of it. On the basis of mechanism, there are three types of air gun, namely spring piston air gun, pneumatic air gun, and compressed gas $\left(\mathrm{CO}_{2}\right)$ air gun (Vanzi 2005; Abhyankar et al. 2018). They have been utilized as a part of chasing, sporting, and fighting. These are easily available in an open market with fewer rules and regulations and also considered relatively safe. Although these have been found to have the potential to cause fatal and grievous injuries such as bone micro-fracture (Kieser et al. 2013), and ocular injuries (Schein et al. 1994) and even may cause death (Lal and Subrahmanyam 1972). It is evident from the literature that air guns (both rifle and pistol) are being used in illicit activities for a long period and in current time also (Burnt and Packey 1979; Harshey et al. 2017; Morgan et al. 2019). Various researchers studied the different aspects of glass and air gun that may be of forensic interest such as wounding potential and behavior of air gun pellet in ballistic gel (DiMaio et al. 1982; Wightman et al. 2010; Hallikeri et al. 2012; Stankov et al. 2013; Ansari and Chakrabarti 2017; Vedrtnam and Pawar 2017; Hsiao and Meng 2018). In firing incidences, glass may also serve as an intermediate object. Therefore, in the light of above facts, this study was performed with the aim to analyze the multiple fractures on a glass pane made by air rifle.

\section{Materials and methodology}

In this work, 3-, 4-, and 5-mm-thick window panes of $1 \mathrm{ft}^{2}$ were chosen. Three- and 5-mm-thick glasses were transparent while a 4-mm-thick glass category involved both privacy and transparent panes. These panes were fixed in a metal frame with the help of clay. The frame was placed perpendicularly to the muzzle of the rifle at a fixed distance of $12 \mathrm{ft}$. A total of 100 samples were test fired with .177" ( $4.5 \mathrm{~mm})$ caliber Air Rifle. Two fires, one after another, were made on the same side of the glass pane at an average distance of 2.5-3.0" between both points of impact in order to analyze the multiple fractures. Table 1 summarizes the details about the used air rifle and round nose pellet.

Fractured panes were measured with the help of a vernier caliper. Figure 2 shows the hole diameter, thickness, and the diameter of the mist zone that served as parameters for the sample analysis. Mean value and the standard deviation of the measurements for the above said parameters were calculated. Statistical operation, i.e., "chi-square test (test of goodness of fit)," was also applied in this series of analysis to check whether the data is consistent or not. Level of significance $(\alpha)$ is the probability of type one error (rejection of null hypothesis when it is true). The null hypothesis $\left(\mathrm{H}_{0}\right)$ is accepted when the calculated value of chi-square is less than the 
Table 1 Specification about the air rifle and pellet

\begin{tabular}{ll}
\hline $\begin{array}{l}\text { Specifications of air rifle } \\
\text { Name of the }\end{array}$ & $\begin{array}{l}\text { Precihole Sports Pvt. Ltd., Thane, } \\
\text { manufacturer }\end{array}$ \\
Model & SX 100 Pegasus \\
Caliber & $0.177 "(4.5 \mathrm{~mm})$ \\
Velocity & $240 \mathrm{mps}$ \\
Power & $16 \mathrm{~J}$ \\
Weight & $3.1 \mathrm{~K} . \mathrm{G}$. \\
Total length & $1065 \mathrm{~mm}$ \\
Barrel length & $450 \mathrm{~mm}$ \\
Specifications of the pellets & \\
Material & Lead \\
Brand & Magnum \\
Caliber & $0.177 "$ (4.5 mm) \\
Average weight of pellet & $0.41 \mathrm{~g}$ for round nose \\
used &
\end{tabular}

Source: SX100 Owner Manual Published by Precihole Sports, Pvt. Ltd., Thane, Maharashtra, India

tabulated value at a particular degree of freedom (for " $n$ " sample, degree of freedom is " $n-1$ ").

\section{Results}

An analysis of the multiple fracture pattern was performed on the basis of measurements made on the fractured pane. In most of the cases, 3-mm thick glass panes broke down on the course of second shot. Table 2 shows the value of mean and standard deviation of the measurements and the description of the chi-square test for the first and second target (T1 \& T2) of 3-mm transparent, 4-mm transparent and privacy, and 5-mm transparent glass panes. The trends in T1 and T2 were studied comparatively. In order to analyze the consistency, chi-square test was applied on the hole diameter of T1 and T2 of all the glass panes. Null hypothesis was rejected (as the calculated value was greater than the tabulated value) only in the case of T2 of $3 \mathrm{~mm}$. A consistency in the hole diameter was observed that has also been statistically proved.

On the visual examination of the fracture glass panes, it was revealed that $3-\mathrm{mm}$ fractured panes possess circular-shaped mist zone. Mist zone of 4-mm (both transparent and privacy) glass panes was found to be elliptical or triangular with rounded edges while mist zone of 5-mm-thick glass panes was found to be irregular.

\section{Discussion}

This study was performed to analyze the multiple glass fracture. From the measurement, it was noted that the hole diameter values lies between 4.1 and $6.7 \mathrm{~mm}$. Previously, Harshey et al. (2017) analyzed the pattern of glass fractures that were made by the air rifle on the glasses of different thickness. In this approach, a special aspect of glass coating with Sun Control Film (SCF) was also taken under consideration. It was found that the hole diameter ranges between 4.77 and $7.5 \mathrm{~mm}$. SCF does not contribute any notable difference in fracture pattern. It is notable that this presented study gave results in a similar manner (reproducibility of the results) as that of pronounced by Harshey et al. (2017) and Abhyankar et al. (2018).

Another extensive approach is also reported by Waghmare et al. (2016). In this study, glasses were fractured by improvised pistol and several regular firearms manufactured in the Indian Ordinance Factory, India. Concerning the analysis of the data, the hole diameter is somewhat doubled to bullet caliber, except in case of a 9-mm pistol. Except in the case of improvised pistol, the values of hole diameter were in between 11.04 and $14.63 \mathrm{~mm}$. Improvised pistol does not show any consistency as its manufacturing is not standardized.

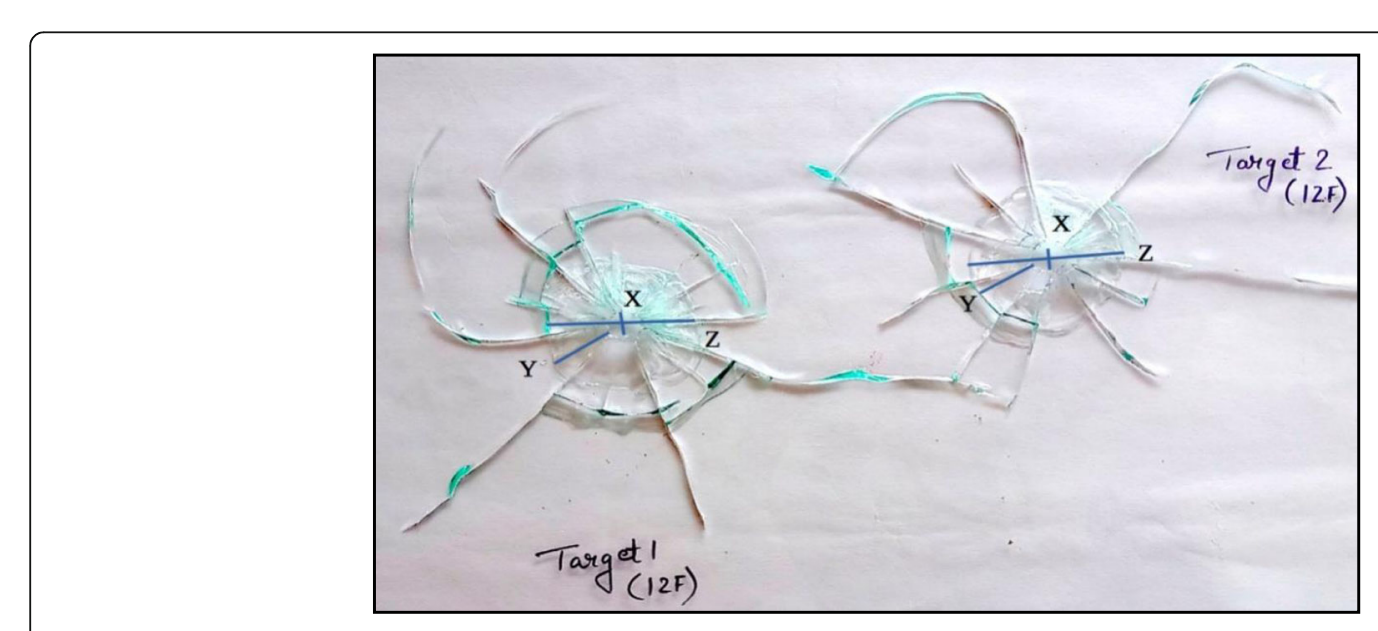

Fig. 2 Representation of the parameters: the hole diameter $(X)$, diameter of mist zone $(Z)$, thickness of mist zone $(Y)$ 
Table 2 Value of mean and standard deviation of the measurements and description of the chi-square test for the T1 and T2 of different glass panes

\begin{tabular}{|c|c|c|c|c|c|c|c|c|c|c|}
\hline \multirow[t]{2}{*}{ Thickness Of Glass } & \multicolumn{2}{|c|}{ Hole diameter } & \multicolumn{2}{|c|}{ Thickness of mist zone } & \multicolumn{2}{|c|}{ Diameter of mist zone } & \multicolumn{2}{|c|}{ Radial fracture count } & \multicolumn{2}{|c|}{ Concentric fracture count } \\
\hline & $\mathrm{T} 1$ & $\mathrm{~T} 2$ & $\mathrm{~T} 1$ & $\mathrm{~T} 2$ & $\mathrm{~T} 1$ & $\mathrm{~T} 2$ & $\mathrm{~T} 1$ & $\mathrm{~T} 2$ & $\mathrm{~T} 1$ & $\mathrm{~T} 2$ \\
\hline \multicolumn{11}{|l|}{ Mean values (in mm) } \\
\hline $3 \mathrm{~mm}$ (transparent) & 5.76 & 1.73 & 12.09 & 4.45 & 28.57 & 9.422 & 11 & 3 & 7 & 2 \\
\hline $4 \mathrm{~mm}$ (transparent) & 6.08 & 5.70 & 12.64 & 12.40 & 31.54 & 28.78 & 9 & 8 & 7 & 9 \\
\hline 4 mm (privacy) & 5.74 & 5.95 & 13.79 & 11.40 & 33.17 & 28.90 & 8 & 7 & 5 & 5 \\
\hline $5 \mathrm{~mm}$ (transparent) & 5.61 & 5.42 & 9.35 & 13.51 & 23.66 & 32.11 & 9 & 8 & 5 & 4 \\
\hline \multicolumn{11}{|l|}{ Standard deviation } \\
\hline $3 \mathrm{~mm}$ (transparent) & 0.37 & 2.77 & 1.01 & 6.69 & 2.33 & 14.04 & - & - & - & - \\
\hline $4 \mathrm{~mm}$ (transparent) & 0.29 & 0.43 & 0.78 & 1.07 & 1.43 & 3.62 & - & - & - & - \\
\hline 4 mm (privacy) & 0.61 & 0.46 & 1.55 & 2.13 & 2.44 & 4.33 & - & - & - & - \\
\hline $5 \mathrm{~mm}$ (transparent) & 0.49 & 0.60 & 2.95 & 4.23 & 6.77 & 9.008 & - & - & - & - \\
\hline \multicolumn{11}{|c|}{ Description of chi-square observations } \\
\hline \multicolumn{11}{|c|}{ Null hypothesis $\mathrm{H}_{0}$ : All the values are consistent, i.e., equals to the mean value. } \\
\hline & \multicolumn{4}{|c|}{ Calculated value of chi-square } & \multicolumn{4}{|c|}{ Tabulate value of chi-square at $5 \%$ level of significance } & & \\
\hline & $\mathrm{T} 1$ & & $\mathrm{~T} 2$ & & & & & & & \\
\hline $3 \mathrm{~mm}$ (transparent) & 0.576 & & 69.022 & & 13.848 & & & & & \\
\hline $4 \mathrm{~mm}$ (transparent) & 0.347 & & 1.99032 & & & & & & & \\
\hline 4 mm (privacy) & 1.560 & & 1.19400 & & & & & & & \\
\hline $5 \mathrm{~mm}$ (transparent) & 1.032 & & 1.619 & & & & & & & \\
\hline
\end{tabular}

The above-discussed studies show various trends of hole diameter made by different firearms. The type of weapon (i.e., whether standard firearm or air gun) could be determined on the basis of hole diameter by which glass was fractured. The pattern of glass fracture also differs in case of different firearms, i.e., the patterns made by air rifle are different from that of caused by regular firearms. Similarity in the patterns may be observed on general visual examination.
Apart from it, this study also provides a means to establish the sequence of shot. In this study, many panes were obtained in which radial cracks of T1 and T2 did not meet each other (Fig. 3). In such condition, the sequence of shot may not be established by the rule of thumb. Results of this study showed that it was found that the hole diameter of $\mathrm{T} 1$ for 4-mm transparent and $5-\mathrm{mm}$ transparent is greater than that of $\mathrm{T} 2$ while the hole diameter of $\mathrm{T} 2$ is greater for 4-mm privacy pane.

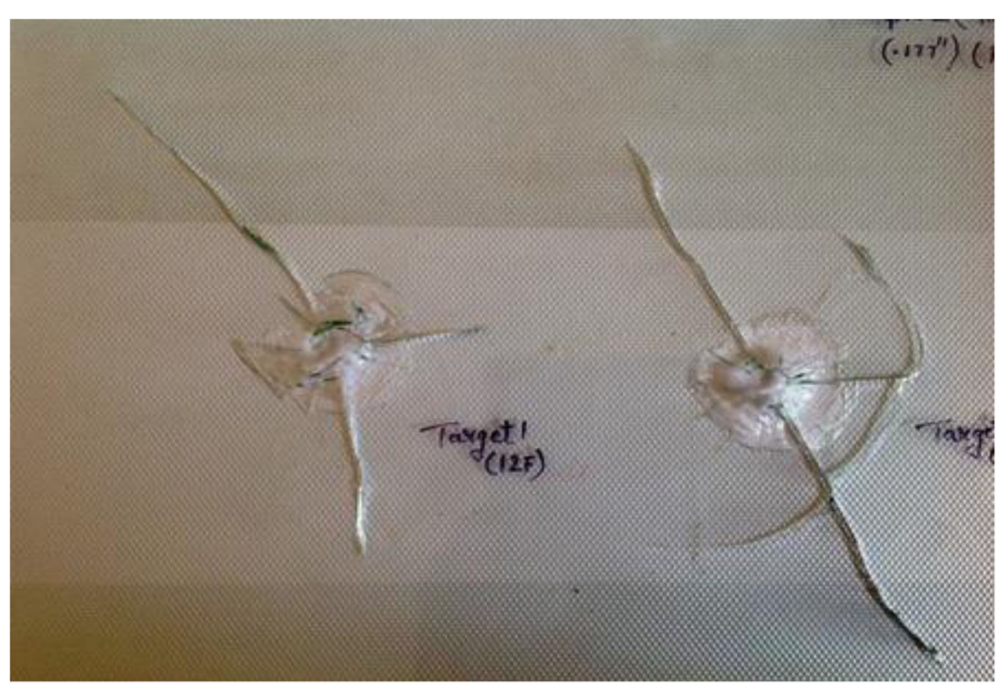

Fig. 3 Multiple fracture on the glass pane 
This may be indicative for the establishment of the sequence of hole.

\section{Conclusion}

This study was performed with the aim to analyze the multiple fracture pattern made by air rifle of .177" caliber. In this study, regularity was observed in the measurements made on fixed parameters. Trends and observations may lead to the determination of the kind of weapon that may play a key role in forensic investigation. It can easily distinguish between the fracture pattern made by regular firearm and the air rifle. The hole diameter value lies in between $4.1 \mathrm{~mm}$ and $6.7 \mathrm{~mm}$. This study also provides another mean to estimate the sequence of hole. This aspect may be explored further. Consistency in the features, trends, and characteristics is found to be decreasing with the increasing thickness of the glass panes. The findings of this work may efficaciously help in the forensic investigation in the course of the scene of crime investigation and to interpret the fractured glass panes.

\section{Acknowledgements}

Not applicable.

\section{Funding}

Not applicable.

\section{Availability of data and materials}

All data generated or analyzed during this study. Raw data is available with one of the authors (NT).

\section{Authors' contributions}

All the authors made significant contributions to all aspects of this research. All authors read and approved the final manuscript.

\section{Ethics approval and consent to participate}

Not applicable.

\section{Consent for publication}

Not applicable.

\section{Competing interests}

The authors declare that they have no competing interests.

\section{Publisher's Note}

Springer Nature remains neutral with regard to jurisdictional claims in published maps and institutional affiliations.

\section{Author details}

${ }^{1}$ Dr. A.P.J. Abdul Kalam Institute of Forensic Science and Criminology, Bundelkhand University, Jhansi, Uttar Pradesh 284128, India. ${ }^{2}$ State Forensic Science Laboratory, Government of NCT, New Delhi, India.

Received: 31 January 2019 Accepted: 23 April 2019

Published online: 17 May 2019

\section{References}

Abhyankar S, Srivastava A, Yadav V, Nigam K, Harshey A (2018) Glass fractures made from different pellet shapes- a preliminary study. Journal of Forensic Science and Criminal investigation 8:1-7. https://doi.org/10.19080/JFSCI.2018. 08.555739

Ansari MM, Chakrabarti A (2017) Influence of projectile nose shape and incidence angle on the ballistic perforation of laminated glass fibre composite plate.
Compos Sci Technol 142:107-116. https://doi.org/10.1016/j.compscitech.2016. 12.033

Burnt GR, Packey AJ (1979) The criminal use of air weapons. Journal Forens Sci Soc 19

Caddy B (2001) Forensic examination of glass and paint. First (ed) Taylor and Francis, London and New York

Copley GJ (2001) The composition and manufacture of glass and its domestic and industrial applications. In: Caddy B (ed) Forensic examination of glass and paint. Taylor \& Francis, New York, pp 27-46

Curran JM, Hicks TN and Buckleton JS (2000) Forensic Interpritation of Glass Evidence.CRC press LLC. Boca Raton London New York Washington, D.C. ISBN 0-8493-0069-X (alk)

DiMaio VJ, Copeland AR, Besant PEM, Fletcher LA, Jones A (1982) Minimal velocities necessary for perforation of skin and air gun pellets and bullets. J Forensic Sci 27:894-898

Gogotsi GA, Mudrik SP (2010) Glasses: new approach to fracture behavior analysis. J Non-Cryst Solids 356:1021-1026

Grady DE (2010) Length scales and size distributions in dynamic fragmentation. Int J Fract 163:85-99

Griffith AA (1920) The phenomena of rupture and flow in solids. A Philosophical Transactions of the Royal Society of London 221:163-198

Hallikeri VR, Gauda HS, Kadagaudar SA (2012) Country made scar gun vs. air gun; comparative study of terminal ballistics using gelatin blocks. Forensic Science Int 214:148-151

Harshey A, Srivastava A, Yadav VK, Nigam K, Kumar A, Das T (2017) Analysis of glass fracture pattern made by .177" $(4.5 \mathrm{~mm})$ caliber air rifle. Egypt $J$ Forensic Sci 7:1-8

Hsiao YT, Meng HH (2018) Evaluation of wounding potential of airguns using aluminium witness plates. Australian Journal of Forensic Sciences. https://doi. org/10.1080/00450618.2018.1553207

Jauhari M, Chatterjee SM, Gosh PK (1974) Remaining velocity of bullets fired through glass plates. J Forensic Science Soci 14:3-7

Kieser CD, Riddel R, Kieser AJ, Theis J, Swain VM (2013) Bone micro-fracture observations from direct impact of slow velocity projectiles. Journal of Archive and Military Medicine 2:1-6

Lal RC, Subrahmanyam BV (1972) Accidental death by air rifle. Forensic Sci 1:441443

Mcijinkins SP, Thornton JI (1973) Glass fracture analysis- a review. Forensic Science 2:1-27 https://doi.org/10.1016/0300-9432(73)90002-2

Mistek E, Fikiet MA, Khandasammy SR, Lednev IK (2018) Toward Locard's exchange principle. Recent developments in forensic trace evidence analysis. Anal Chem. https://doi.org/10.1021/acs.analchem.8b04704

Morgan A, Aqil NA, Okeil NA, Ghaleb SA, Otaibi AF, Alashqar HM, Ghuwainem SOA, Qahtani MAM (2019) Firearm injuries in rural Saudi Arabia: incidence, patterns, management, and cost. Egypt J Forensic Sci 9:1-7

Mott NF (1964) Brittle fracture in mild steel plates. Engineering 165:16-18

Overend M, Gaetano SD, Haldimann M (2007) Diagnostic interpretation of glass failure. Struct Eng Int (2):151-158

Robertson J, Roux C (2010) Trace evidence: Here today, gone tomorrow? Sci Justice 50:18-22

Schein OD, Enger C, Tielsch JM (1994) The context and consequences of ocular injuries from air guns. American J of Ophthalmo 117:501-506

Stankov A, Jakovski Z, Pavlovski G, Muric N, Dwork AJ (2013) Air gun injury with deadly aftermath- case report. Legal Med 15:35-37

Stoney DA, Thornton JI (1985) The forensic significance of the correlation of density and refractive index in glass evidence. Forensic Sci Int 29:147-157

Vanzi M (2005) Pellet guns and BB guns: dangerous playthings in the open market. California Senate Office of Research, California, http://sor.senate.ca. gov/sites/sor.senate.ca.gov/files/Pellet\%20Guns\%20and\%20BB\%20Guns.pdf

Vedrtnam A, Pawar SJ (2017) Laminated plate theories and fracture of laminated glass plate- a review. Eng Fract Mech 186:316-329

Waghmare NP, Lal A, Anand VR (2016) Investigation of forensic glass Fractography made by different ammunition. Austin Journal of Forensic Science and Criminology 3:1-5

Waghmare NP, Manna A, Rao MS, Sen PK (2003) Statistical analysis of impact parameters of soft nose bullet fired on windowpane. Forensic Sci Int 135: 181-187

Wightman G, Beard J, Allison R (2010) An investigation into the behavior of air gun pellets in ballistic gel and their interaction with bone. Forensic Science Int. 200:41-49 